\title{
A New Method for the Cell Culture of Anaerobic Bacteria: P. Gingivalis- or P. Endodontalis-Mediated Pathways in RAW264.7 Cells
}

\author{
Xiaoren Tang ${ }^{1 *}$, Nujud Zayed H Alamry ${ }^{2}$, Yinian Tang ${ }^{3}$ and Serge Dibart ${ }^{1}$ \\ ${ }^{1}$ Department of Periodontology, Boston University Goldman School of Dental School, USA \\ ${ }^{2}$ Princess Nourah Bint Abdulrahman University, Riyadh, Saudi Arabia \\ ${ }^{3}$ Boston University Medical School USA
}

Received: June 12, 2018; Published: June 19, 2018

*Corresponding author: Xiaoen Tang, Department of Periodontology, Boston University Goldman School of Dental School, 650 Albany Street, Boston, MA 02118, USA

Abstract

The growth of gram-negative anaerobes requires the complete absence of oxygen ( $\nabla 0.001 \%)$, and is usually treated in an anaerobic chamber.However, this traditional method is costly and time consuming.In this study, we describe a new, rapid method for the culture of anaerobic bacteria, such as $P$. gingivalis, and $P$. endodontalis, in a rotary shaker under anaerobic conditions, by sealing the bacteria growth media from oxygen exposure using a mineral oil overlay. By using these cells, we found P. gingivalis or $P$. endodontalis mediated different signaling pathways in RAW264.7 cells.

Keywords: Kinases; Phosphorylation; Menadione; Centrifugation; Proliferation; Adult Periodontitis; Anaerobic Bacteria; Anova; Periodontal Pathogens; Phosphorylation

\section{Introduction}

It is known that anaerobicbacteriasuch as $P$. gingivalis [1] is involved in the progression and initiation of multiple forms of periodontal disease such as adult periodontitis [2,3]. However, it takes a long time (about 72 hours) to reach optimal cell density.This study will elaborate upon a new, rapid and less expensive method for the culture of anaerobes, such as $P$. gingivalis and $P$. endodontalis. Further analysis by western blot confirmed that rapid culture does not affect cell's biological functions. In addition, we found three different $P$. gingivali or $P$. endodontalis-mediated pathways via specific regulation of kinases involved in phosphorylation. Similar treatment of these cells upregulated the expression of certain transcriptional factors as well. Additionally, this method can be utilized beyond periodontal pathogens for research with most anaerobic bacteria.

\section{Materials and Methods}

Cells: The bacterial strains, $P$ gingivalis (ATCC 33277) and $P$. endodontalis (ATCC 27067) were grown either in brain-heart infusion broth (BHI), $5 \mathrm{mg} / \mathrm{l}$ hemin and $1 \mathrm{mg} / \mathrm{l}$ menadione under the anaerobic chamber, unshaken, at $37^{\circ} \mathrm{C}$, or grown in the same medium with/without a mineral oilat a ratio of 1:20 (mineral oil: medium) at $220 \mathrm{rpm}$ in a rotary shaker at $37^{\circ} \mathrm{C}$. The cells were collected by centrifugation at the indicated time points, washed and resus pended in BSM. The bacterial cell density was measured at $600 \mathrm{~nm}$ $\left(\mathrm{OD}_{600}\right)$. RAW264.7 cells (TIB 71, ATCC) were cultured in a RPMI1640 media (Cat\#: 11875-093, Life Technologie s, NY) with $10 \%$ $\mathrm{FBS}$ at $37^{\circ} \mathrm{C}$ in a $5 \% \mathrm{CO} 2$ atmosphere.

Elisa: The media from the treated RAW264.7 cells was used for an ELISA assay with TNF- $\alpha$ detection (kit: Cat\#:KMC30110, Invitrogen). ELISA immunoreactivity was quantified using a microplate reader (Model 680, Bio-Rad). The data was analyzed and then graphed.

Western Blot Analysis: Pre-cultured RAW264.7 cells were untreated or treated with $1 \times 10^{5}$ P. gingivalis or P. endodontalis cells overnight. The proteins purified from each group were detected by western blotting, following the manufacturer's instructions. Antibodies were purchased from the following vendors: LITAF (611615) from BD Biosciences, p38 (sc-81621), p-p38 (sc-166182), ERK1 (sc-271270), p-ERK1 (sc101760), JNK (sc-572), p-JNK (sc-81502), PI3K (sc-365290) and actin (C-11) from Santa Cruz Biotechnology, p-PI3K (42285), AKT (92725), p-AKT (13038), c-Jun (2315) and p-c-Jun (3270) from Cell Signaling.

Statistical Analysis: All experiments were performed in triplicate and statistical analyses were conducted with the SAS software package. All data were normally distributed. For multiple mean 
comparisons, we conducted analysis of variance (ANOVA), while for single mean comparison, we used the Student's t-test. For the time-course study, we used a two-way repeated measure ANOVA. P values less than 0.05 were considered significant.

\section{Results}

To test the effectiveness of our proposed method (described in this study) in promoting the in-vitro culture of anaerobic bacteria, P. gingivalis was cultured under three different conditions. One was to culture the cells in the growing liquid medium in an orbital shaker without the addition of mineral oil to bacterial medium. Another condition was to culture cells in the anaerobic chamber as described [4]. Lastly, cells were cultured while shaking with an appropriate volume of mineral oil on top of the liquid medium. As shown in Figure 1A, no increase in the cell number at any time frame could be noticed under the first culture condition due to the exposure of the bacterial anaerobes to oxygen. The number of anaerobes detectable in media with the overlay of mineral oil (condition 3) rapidly increased after 4-hours of incubation and peaked around 24-hours.Although the bacterial cell density reached an OD600冈1 under the second culture condition and in the anaerobic chamber, it took $72 \mathrm{hrs}$ to grow. Therefore, it seems clear that the new anaerobic culture method is more efficient and results in anaerobe proliferation close to three times faster than the traditional manner in the anaerobic (cell) chamber.
In order to check if this proposed method could be useful for growing different anaerobic pathogens, other periodontal pathogens, $P$. endodontalis, and P. gingivalis as a control, were randomly selected and cultured by the new method. As shown in Figure $1 \mathrm{~B}$, all anaerobes have shown a similar pattern of increase in cell number (OD600冈0.7) after $24 \mathrm{hrs}$ of cell culture when grown using the new method (condition 3). Furthermore, we were interested in observing whether the rapid-grown anaerobic cells would still have their natural biological function. Thus, a western blot analysis was performed. As shown in Figure 2, The RAW264.7 cells treated by P. gingivalis or P. endodontalis showed a variety of phosphorylation levels for the kinases, which were separated into three groups, group 1: both protein and phosphorylation level of kinase (p38, ERK1 \& JNK for P. gingivalis, or p38 \& ERK1 for P. endodontalis) were increased; group 2: only phosphorylation level of the kinase (PI3K \& Akt for P. gingivalis, c-Jun for P. endodontalis) was increased; group 3: neither protein level nor phosphorylation level of the kinase (c-Jun for P. gingivalis, PI3K, Akt \& JNK for P. endodontalis) was increased. Meanwhile, we found that the treatment of cells by $P$. gingivalis or P. endodontalis induced the transcriptional factor such as LITAF or NFkB gene expression. Overall, this result suggests that the new method is a rapid, effective, and efficient method not only for culturing periodontal pathogens but also anaerobic bacteria in general.

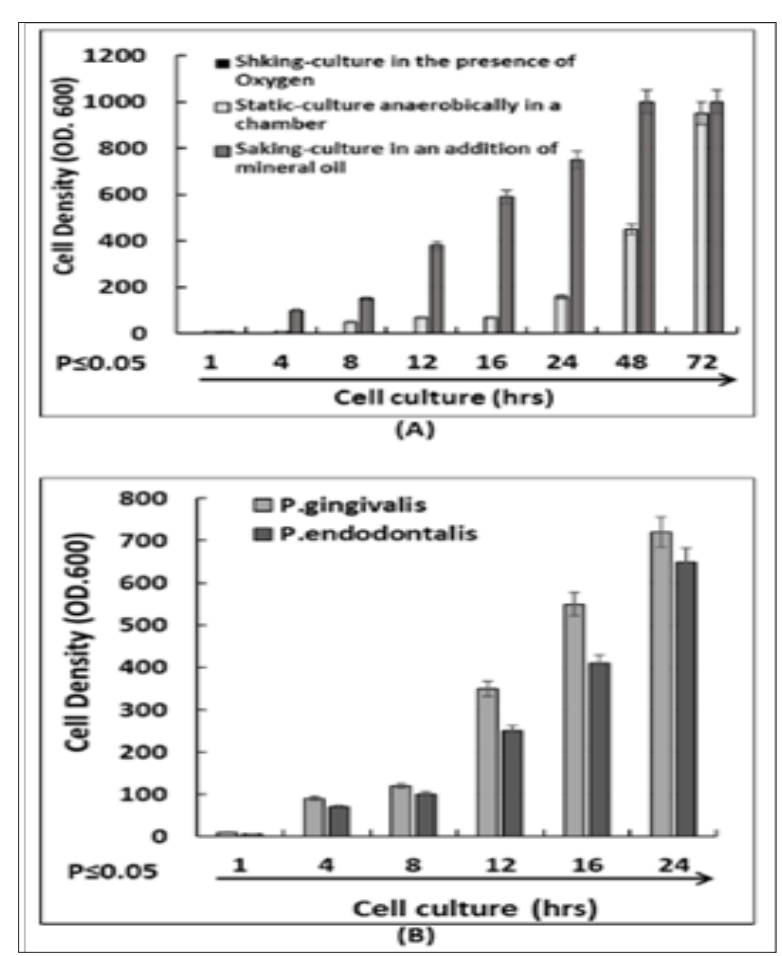

Figure 1: The measurement of $P$. gingivaliscell density (OD600). (A) P. gingivalis were grown and obtained under three different conditions: 1 . Cells were cultured in the liquid growing medium without an addition of mineral oil on a rotary shaker; 2 . Cells were static-cultured under the anaerobic chamber. 3. Cells were cultured in the liquid growing medium in an addition of mineral oil on a rotary shaker. (B) The measurement of $P$. gingivalis, $P$. intermedia or $P$. endodontalis cell density $\left(\mathrm{OD}_{600}\right)$. The cells were grown in the liquid medium in an addition of mineral oil on a rotary shaker and obtained from different time-points. The triplicate measurements were performed and graphed. 


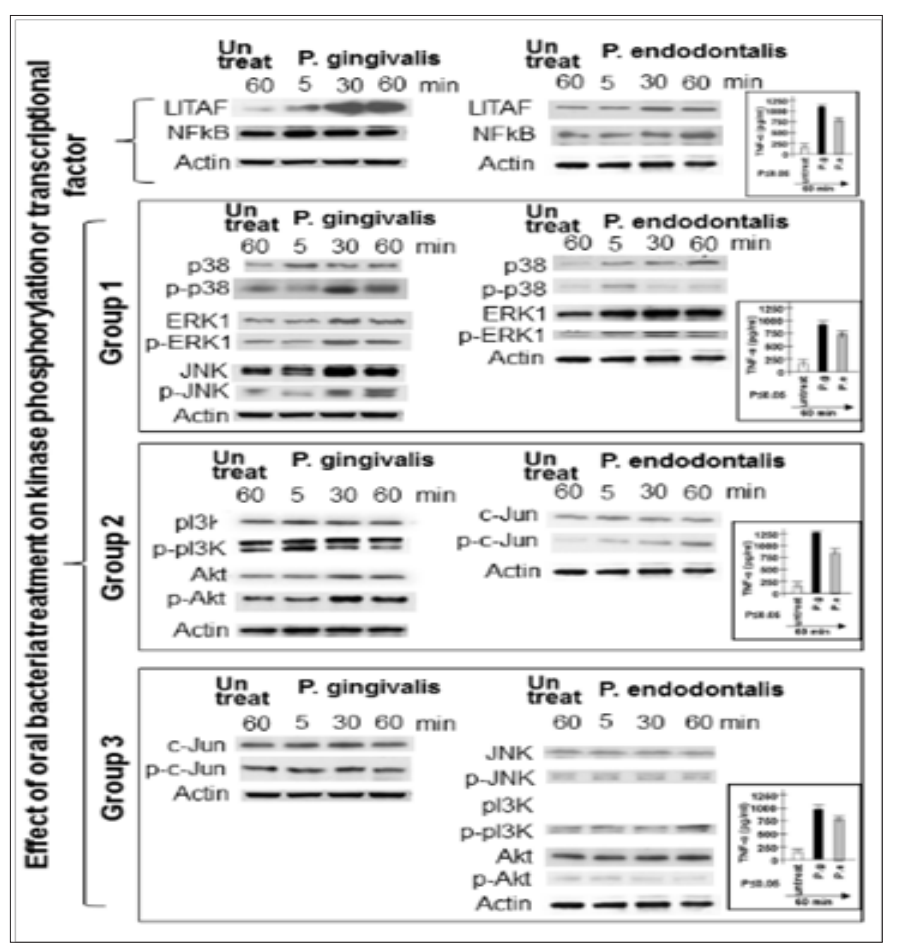

Figure 2: Analysis of phosphorylated kinases in response to $P$. gingivalis or $P$. endodontalis treatment in RAW264.7 cells. To evaluate if a response by $P$. gingivalis or $P$. endodontalis caused an effect on kinases/factors, a western blot was performed. Pre-cultured RAW264.7 cells were untreated as control or treated with $1 \times 105$ cells $/ \mathrm{ml} P$. gingivalis or P. endodontalis as the test group for 5, 30, or 60min. The medium from each group was used for ELISA and its results was graphed and attached. Meanwhile, cell extractions were purified and their protein levels or phosphorylation levels were identified by western blot, which were then compared to the control (Actin). The intensities of untreated cells were assigned to a base value (100\%). Intensity of other test groups was calculated relative to this base value. Triplicate assays above were conducted. Mean SEM.

\section{Discussion}

It has been well established that periodontal diseases can be induced through infection with its respective pathogen, specifically, $P$. gingivalis.Furthermore, it is understood that $P$. gingivalis is incapable of proliferation and its survival exponentially declines when directly exposed to oxygen, even at extremely low levels $(\nabla 0.01 \%$, data not shown).We were thus interested in determining how to mimic optimal anaerobic conditions for the P. gingivalis cell culture to more easily obtain a larger volume of cells for study. In this paper, we report that an overlay of mineral oil is sufficient to protect the anaerobic periodontal pathogens grown in-vitro in liquid media from exposure to environmental oxygen and the subsequent inhibitory effects on bacterial growth and proliferation. We demonstrate that these cells can be cultured at $220 \mathrm{rpm}$ in a rotary shaker that effectively reduces the time required for the proliferation of these anaerobes in comparison to other commonly used methods.

\section{References}

1. Hope CK, HindleyJA, Khan Z, de Jong Ede J, Higham S, et al. (2013) Lethal photosensitization of Porphyromonas gingivalis by their endogenous porphyrins under anaerobic conditions: an in vitro study. Photodiagnosis Photodyn Ther10(4): 677-682.

2. Al bandar JM (2014)Aggressive and acute periodontal diseases. Periodontol 65(1): 7-12.

3. Bamea TV,Sava A, Gentimir C, Goriuc A, Boișteanu O, et al.(2015) Genetic polymorphisms of TNFA and IL-1A and generalized aggressive periodontitis. Rom J MorpholEmbryol 56(2): 459-464.

4. Kikuchi Y, Sasaki H, Furuhata Y, Tazawa Y, Horiuchi H, et al. (2007) Evaluation for anaerobic culture system: Anoxomat Mart II.RinshoBiseibutshuJinsokuShindanKenkyukai Shi 18(1): 11-17.

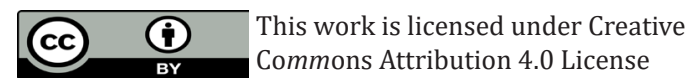

Submission Link: https://biomedres.us/submit-manuscript.php

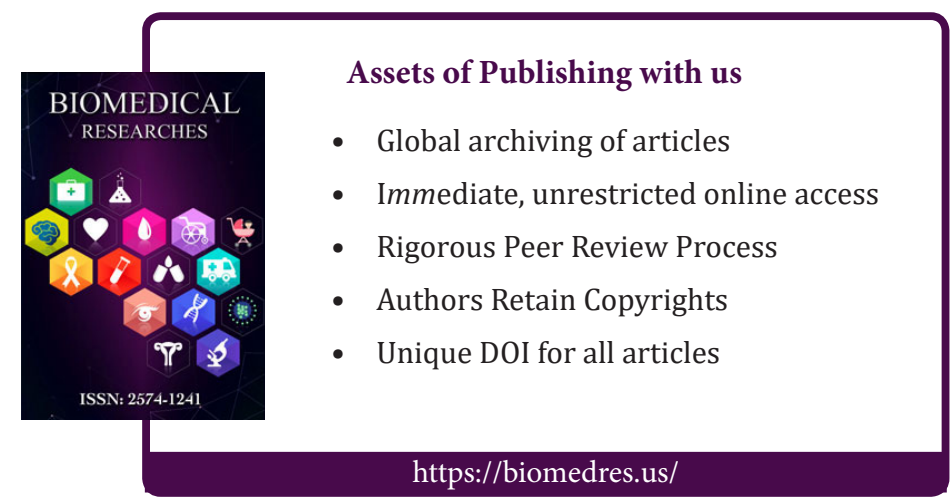

